\title{
ON THE PRODUCTS OF WEAKLY LINDELÖF SPACES
}

\section{A. HAJNAL AND I. JUHÁSZ}

ABSTRACT. The aim of this note is to show, without using any special set-theoretic assumptions, that the product of two (weakly) Lindelöf spaces is not necessarily weakly Lindelöf.

In [1] M. Ulmer has constructed two weakly Lindelöf spaces whose product is not so; in his construction, the assumption $2^{\boldsymbol{N}_{0}}=2^{\boldsymbol{N}_{1}}$ was essentially employed. In this short note we shall provide another such example (where the factors are even Lindelöf), in the construction of which no additional set-theoretic assumption is used.

To start with, we shall deal with some properties of the "half-open" topologies on linearly ordered sets which may be interesting in themselves. We recall that, given a cardinal number $\alpha, X$ is (weakly) $\alpha$-Lindelöf if every open cover of $X$ has a subcover (weak subcover, i.e. a subfamily whose union is dense in $X$ ) of cardinality $\leq a$.

Let $\langle R, \prec\rangle$ be a linearly ordered set. We shall denote by $R^{+}$and $R^{-}$, respectively, the spaces on $R$ for which the half-open intervals of the form $[x, y)$ and $(x, y]$, respectively, form an open basis.

Lemma 1. Let $a$ be an infinite cardinal number, and let $\langle R,\langle\rangle$ be an order complete linearly ordered set in which there is no decreasingly or increasingly ordered subset of type $\alpha^{+}$. Then both $R^{+}$and $R^{-}$are a-Lindelöf spaces.

Proof. It will obviously suffice to show that $R^{+}$is $\alpha$-Lindelöf. To see this, let $\mathcal{U}$ be a cover of $R^{+}$by basic open sets of the form $[x, y)$. First we claim that for any $a, b \in R, a \prec b$, the segment $[a, b]$ can be covered by at most $a$ members of $\mathcal{U}$.

Indeed, using the completeness of $\langle R, \prec\rangle$, there is a $c \in[a, b]$ which is the least upper bound of those $d \in[a, b]$ for which the segment $[a, d]$ can be covered by $\leq a$ members of $\mathcal{U}$. We claim that $c=b$.

Received by the editors October 16, 1973.

AMS (MOS) subject classifications (1970). Primary 54B10, 54D20.

Key words and phrases. Product space, Lindelöf, weakly Lindelöf. 
Suppose, on the contrary, that $c<b$. The segment $[a, c]$ itself can be covered by $\leq a$ members of $\mathcal{U}$. This is obvious if $c=a$ or if $c$ has an immediate predecessor. If not, it follows from the second condition on $\langle R,\langle\rangle$. Indeed, in this case we can take an increasing, well-ordered sequence $\left\langle c_{\xi}, \xi<\lambda\right\rangle$ converging to $c$ from below, with $c_{\xi} \in(a, c)$ and $\lambda \leq \alpha$. By the choice of $c$, every segment $\left[a, c_{\xi}\right]$ can be covered by $\leq a$ members of $\mathcal{U}$; hence, so can $[a, c)=\bigcup\left\{\left[a, c_{\xi}\right]: \xi<\lambda\right\}$, and $[a, c]$ as well.

Now there are two cases to be distinguished.

First, if $c$ has an immediate successor, say $c^{\prime}$, and $[x, y)$ is a member of $\mathcal{U}$ containing $c^{\prime}$, then adding $[x, y)$ to any cover of $[a, c] w$ ith $\leq a$ members of $\mathcal{U}$ we obtain such a cover of $\left[a, c^{\prime}\right]$, contradicting the choice of $c$. Similarly if $c$ has no immediate successor and $[x, y)$ is a member of $\mathcal{U}$ containing $c$, then $[x, y)$ contains a $c^{\prime}>c$, hence, adding it to an appropriate cover of $[a, c]$, we again get a contradiction.

Now making use again of the second condition on $\langle R,\langle\rangle$, we can obtain sequences $\left\langle a_{\xi}: \xi\langle a\rangle\right.$ and $\left\langle b_{\xi}: \xi<a\right\rangle$ such that the first one is coinitial and the second is cofinal in $\langle R,\langle\rangle$. According to what we have proved above, every segment $\left[a_{\xi}, b_{\eta}\right]$ can be covered by $\leq a$ members of $\mathcal{U}$, hence so can

$$
R^{+}=\bigcup\left\{\left[a_{\xi}, b_{\eta}\right]: \xi, \eta<a\right\} .
$$

This completes the proof.

Lemma 2. Let $\langle R,\langle\rangle$ be a densely ordered set with $d(R)\rangle \beta$ (i.e., $R$ does not contain a dense subset of cardinality $\leq \beta)$. Then the product space $R^{+} \times R^{-}$is not weakly $\beta$-Lindelöf.

Proof. Let us denote, as usual, by $\Delta$ the diagonal $\Delta=\{\langle p, p\rangle: p \in R\}$ of the product $R^{+} \times R^{-}$, and put

$$
\Gamma=\left\{\langle p, q\rangle \in R^{+} \times R^{-}: p \prec q\right\} \text {. }
$$

First we show that $\Gamma$ is open in $R^{+} \times R^{-}$. Indeed if $p \prec q$ then, by the denseness of $\langle R,\langle\rangle$, there is an $r$ with $p<r<q$, and obviously $[p, r) \times$ $(r, q]$ is a neighbourhood of $\langle p, q\rangle$ contained in $\Gamma$.

Thus

$$
\mathcal{U}=\{\Gamma\} \cup\{[p, \rightarrow) \times(\leftarrow, p]: p \in R\}
$$

is an open cover of $R^{+} \times R^{-}$, since for any $\langle p, q\rangle$ with $p \succeq q$ we have

$$
\langle p, q\rangle \in[p, \rightarrow) \times(\leftarrow, p] \text {. }
$$

We claim that for any subfamily $\bigcirc \subset \mathcal{U}$ with $|\mathcal{O}|=\beta$, the union $V=\bigcup \mathcal{O}$ is 
not dense in $R^{+} \times R^{-}$. Indeed, let us put

$$
A=\{p \in R:[p, \rightarrow) \times(\leftarrow, p] \in \mathcal{O}\} \text {. }
$$

Then $|A| \leq|O|=\beta<d(R)$, so there is an open interval $(a, b)$ of $R$ such that $A \cap(a, b)=\varnothing$; and by the denseness of $\langle R, \prec\rangle$, there is a $c$ with $a \prec c \prec$ $b$.

Now the set $[c, b) \times(a, c]$ is obviously a nonempty open subset of $R^{+} \times R^{-}$, and for any $p \in A$ we have

$$
[p, \rightarrow) \times(\leftarrow, p] \cap[c, b) \times(a, c]=\varnothing,
$$

since either $p \geq b$ or $p \preceq a$. Moreover, we have, trivially,

$$
\Gamma \cap[c, b) \times(a, c]=\varnothing .
$$

This indeed shows

$$
V \cap[c, b) \times(a, c]=\varnothing,
$$

so that $V$ is not dense; thus $R^{+} \times R^{-}$has an open cover with no weak subcover of cardinality $\leq \beta$, and consequently $R^{+} \times R^{-}$is not weakly $\beta$ Lindelöf.

Now we are ready to present our Example.

Example. Let $\langle R, \prec\rangle$ be any linearly ordered set satisfying the following conditions:

(i) $\langle R, \prec\rangle$ is continuously (i.e. both densely and completely) ordered;

(ii) $\langle R,\langle\rangle$ contains no uncountable decreasing or increasing wellordered subset;

(iii) $d(R)=2^{\boldsymbol{N}_{0}}$.

(The unit square with the lexicographic ordering is such an ordered set.) Then $R^{+}$and $R^{-}$are Lindelöf spaces such that $R^{+} \times R^{-}$is not weakly Lindelöf, nor even weakly $\beta$-Lindelöf for any $\beta<2 \boldsymbol{N}_{0}$.

The proof is obvious from Lemmas 1 and 2 .

\section{REFERENCE}

1. M. Ulmer, Products of weakly $\mathrm{K}$-compact spaces, Trans. Amer. Math. Soc. 170 (1972), 279-284.

MATHEMATICAL INSTITUTE OF THE HUNGARIAN ACADEMY OF SCIENCES, H1053, BUDAPEST, REÁLTANODA-U 13-15 HUNGARY

DEPARTMENT OF MATHEMATICS, UNIVERSITY OF WISCONSIN-MADISON, MADISON, WISCONSIN 53706 